\title{
Pollen storages in nests of bees of the genera Partamona, Scaura and Trigona (Hymenoptera, Apidae)
}

\author{
André Rodrigo Rech ${ }^{1} \&$ Maria Lúcia Absy²
}

\begin{abstract}
'Programa de Pós-Graduação em Ecologia (PPGECO), Instituto de Biologia (IB), Universidade Estadual de Campinas (UNICAMP), Rua Monteiro Lobato 970, Caixa Postal 6190, 13083-970 Campinas-SP, Brasil. arech2003@yahoo.com.br.

${ }^{2}$ Coordenação de Pesquisa em Botânica (CPBO), Instituto Nacional de Pesquisas da Amazônia (INPA), Avenida André Araújo 2936, Caixa Postal 478, 69060-001 Manaus-AM, Brasil.
\end{abstract}

\begin{abstract}
Pollen storages in nests of bees of the genera Partamona, Scaura and Trigona (Hymenoptera, Apidae). Bees and angiosperms established a mutualistic relationship along the evolutionary time. The aim of this study is to contribute for the understanding of this relation analyzing pollen stored by stingless bees colonies distributed along the Rio Negro. Fourteen species of Meliponini from the genera Partamona, Scaura, and Trigona were studied with regard to the content of pollen pots. The pollen material was removed from the pollen pots, homogenized, and prepared according to the usual acetolysis technique. The overlap of the trophic niche and the grouping of species by similarity of niches was calculated. The identification revealed 78 pollen types belonging to 36 families, being 37 types attractive and 16 considered as promoters of a temporary specialization event. With the results, it was possible to indicate a list of important plants for meliponiculture in the Amazon.
\end{abstract}

KEYWORDS. Pollination, pollinic resource, stingless bee

RESUMO. Pólen estocado nos ninhos de abelhas dos gêneros Partamona, Scaura e Trigona (Hymenoptera, Apidae). Abelhas e plantas estabeleceram ao longo do tempo evolutivo uma relação mutualística. Buscando contribuir para o entendimento dessa relação, foi analisado o pólen estocado por colônias de abelhas-sem-ferrão distribuídas ao longo do rio Negro. Foram estudados potes de pólen de 14 espécies de Meliponini dos gêneros Partamona, Scaura e Trigona. O material polínico foi retirado dos potes de pólen, homogeneizado e preparado segundo técnica usual de acetólise. Foram calculados a sobreposição de nicho trófico e o agrupamento das espécies pela similaridade de nichos. Foi identificado o total de 78 tipos polínicos, pertencentes a 36 famílias, sendo 37 destes, considerados atrativos, enquanto 16 foram promotores de eventos de especialização temporária. Com os resultados obtidos foi possível indicar uma lista de plantas de importância para a meliponicultura na Amazônia.

PALAVRAS-CHAVE. Abelhas-sem-ferrão, polinização, recurso polínico.

The perpetuation of angiosperms occurs largely due to pollination mechanisms, especially in tropical environments, where most species of flowering plants depend on biotic pollen vectors that promote gene flow, contributing for the formation of viable seeds (Bawa et al. 1985a, b; Bawa 1990; Ennos 1994). In this context, bees stand out as the major group of potential pollinators (Simpson \& Neff 1981). The relationship between bees and angiosperms is very close because pollen and nectar are vital resources for the survival and reproduction of the bees and thus require them to constantly visit flowers throughout their entire lives.

Considering the interaction networks that are established between plants and bees, the study of the identity of each species involved in the interaction may determine a pathway that adds to our understanding of the dynamics of earth's ecosystems (Biesmeijer et al. 2005; Ebeling et al. 2008). One way to access identities in a plant-pollinator interaction network is to make direct observations in the natural conditions of the floral visitors of a given group of plants (Pedro \& Camargo 1991; review in Biesmeijer et al. 2005). However, given the large number of plant species and the geographical breadth of environments such as rainforests, the use of indi- rect methods becomes more relevant. Pollen analysis is one such indirect method, and it has been shown to provide a fairly consistent means of identifying the plants visited by bees (Ramalho et al. 1991; Imperatriz-Fonseca et al. 1993). In spite of the usefulness and versatility of this method, only a few studies of this nature have been conducted to date in the Amazon region (Absy \& Kerr 1977; Absy et al. 1980, 1984; Engel \& Dingemans-Bakels 1980; Kerr et al. 1986, Marques-Souza et al. 1995, 1996, 2002, 2007; Thiele \& Inouye 2007; Marques-Souza 2010).

Once the floral visitors of a given plant species are known, it becomes possible to test their effectiveness in the pollination process and thus increase fruiting or reduce the risk of extinction of endangered species (Kevan \& Imperatriz-Fonseca 2002). For the bees, an understanding of their main pollen sources may allow us to establish plantations that provide them with pollen and consequently lead to the stability and productivity of colonies (Kerr et al. 1986). Furthermore, the knowledge and understanding of the local flora capable of supporting beekeeping activities decreases the need to introduce exotic species and makes beekeeping (with native bees) a practice that is both ecologically correct and potentially sustainable. 
Thus, the aim of this study was to utilize the pollen analysis method to identify the plants used by fourteen species of Meliponini that occur along the Rio Negro channel in Amazonas, Brazil.

\section{MATERIAL AND METHODS}

The pollen material used in this study was collected and kindly provided for this study by a team (M. Mazucato and S. R. M. Pedro) led by Dr. João Maria Franco de Camargo (in memoriam) during a collection expedition held between July 15 and August 15, 1999 (rainy season). The natural nests from which the samples were obtained (pollen pots) were located along an approximately $1600 \mathrm{~km}$ stretch of the Rio Negro and its tributaries located between the Amazonian municipalities of Manaus and São Gabriel da Cachoeira (Fig. 1). The species studied, the locations for the collection of pollen pots, their geographical coordinates and the number of pots used for each species are listed in Table I.

Each pollen pot was considered as one sample. Pollen samples were extracted directly from storage closed pots collected in the natural nests of the bee species listed in Table I. Pollen extraction from pollen storage pots was done using
$5 \mathrm{~mm}$ diameter sterile straws. The whole content of the pollen pots were homogenized with spatulas in Petri dishes, afterwards the samples were weighed and $0.5 \mathrm{~g}$ of each sample was stored in a test tube containing $3 \mathrm{ml}$ Acetic Acid. After sufficient time, the samples were chemically processed via acetolysis (Erdtman 1960). Slides were prepared by mounting the samples in glycerin-gelatin and sealing them with paraffin. Three slides were prepared for each sample. For some bee species, different sample weights were taken from each pollen pot. Test results indicated no significant difference in the richness of pollen types weighing $0.5 \mathrm{~g}$ or above. Furthermore, when new types were found in samples with heavier weights, they were characterized by an extremely low abundance of pollen. For this reason, we decided to use the sample weight of $0,5 \mathrm{~g}$ for each pollen pot.

Pollen types were determined by comparing them to slides from the pollen collection of the Palynology Laboratory of the Instituto Nacional de Pesquisas da Amazônia (INPA) and by consulting the specialized literature. Six hundred pollen grains were counted per sample to figure out their relative abundance. We followed Ramalho et al. (1985) in defining a minimum representation of $10 \%$ to consider a plant as attractive to a given bee species. To define the occurrence of

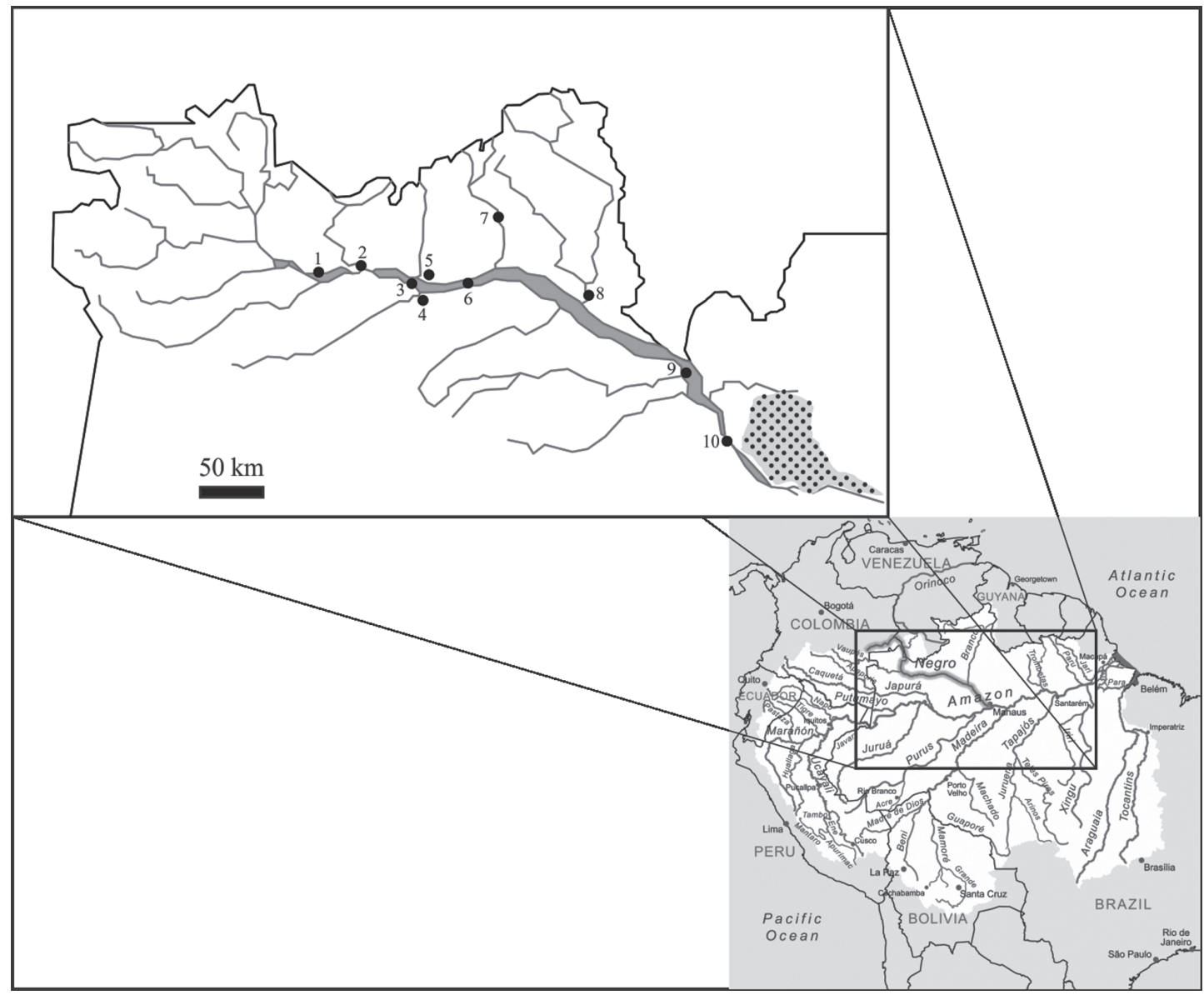

Fig. 1. Schematic map of sample sites along the Rio Negro channel, Amazonas - Brazil. Black dots correspond to sample sites (1. Tapurucuara-Mirim, 2. Ponta Camucuri, 3. Tabocal-Rio Uneiuxi, 4. Nazaré do Rio Negro, 5. Sta. Isabel do Rio Negro-Igarapé do Dará, 6. Samauma-Rio Dará, 7. Foz do Padauari, 8. Rio Demeni-“Pai Raimundo", 9. Lago Caurés and 10. Igarapé Açu-São Francisco) and the dotted area correspond to Manaus city. 
Table I. Bee species, location and geographical coordinates of studied nests and the number of pollen pots studied in Amazonas, Brazil.

\begin{tabular}{|c|c|c|c|c|}
\hline Nest & Species & Locality & Coordinate & Pollen pots \\
\hline 1 & Partamona ailyae Camargo, 1980 & Lago Caurés & $01^{\circ} 18^{\prime} 59^{\prime \prime} \mathrm{S}, 62^{\circ} 11^{\prime} 02^{\prime \prime} \mathrm{W}$ & 1 \\
\hline 2 & Partamona ferreirai Pedro \& Camargo, 2003 & Idem & Idem & 1 \\
\hline 3 & Partamona ferreirai Pedro \& Camargo, 2003 & Nazaré do rio Negro & $0^{\circ} 31^{\prime} 22^{\prime \prime} \mathrm{S}, 65^{\circ} 04^{\prime} 07^{\prime \prime} \mathrm{W}$ & 6 \\
\hline 4 & Partamona mourei Camargo, 1980 & Idem & Idem & 6 \\
\hline 5 & Partamona ferreirai Pedro \& Camargo, 2003 & Ponta Camucuri, Margem Direita & $0^{\circ} 20^{\prime} 02^{\prime \prime} \mathrm{S}, 65^{\circ} 59^{\prime} 20^{\prime \prime} \mathrm{W}$ & 3 \\
\hline 6 & Partamona mourei Camargo, 1980 & Rio Demeni, "Pai Raimundo" & $0^{\circ} 24^{\prime} 01^{\prime \prime} \mathrm{S}, 62^{\circ} 53^{\prime} 41^{\prime \prime} \mathrm{W}$ & 6 \\
\hline 7 & Partamona pearsoni (Schwarz, 1938) & Samauma, rio Daraá & $0^{\circ} 27^{\prime} 7^{\prime \prime} \mathrm{S}, 64^{\circ} 45^{\prime} 35^{\prime \prime} \mathrm{W}$ & 1 \\
\hline 8 & Partamona ailyae Camargo, 1980 & Santa Helena, Ponta do Gavião, rio Negro & $01^{\circ} 23^{\prime} 33^{\prime \prime S}, 61^{\circ} 47^{\prime} 06^{\prime \prime} \mathrm{W}$ & 6 \\
\hline 9 & Partamona mourei Camargo, 1980 & Santa Izabel do Rio Negro, Igarapé Dará & $0^{\circ} 25^{\prime} 04^{\prime \prime S}, 65^{\circ} 01^{\prime} 07^{\prime \prime} \mathrm{W}$ & 1 \\
\hline 10 & Partamona epiphytophila Pedro \& Camargo, 2007 & Tapurucuara-Mirim & $0^{\circ} 25^{\prime} 17^{\prime \prime} \mathrm{S}, 66^{\circ} 24^{\prime} 22^{\prime \prime} \mathrm{W}$ & 5 \\
\hline 11 & Partamona ailyae Camargo, 1980 & Idem & Idem & 6 \\
\hline 12 & Partamona ferreirai Pedro \& Camargo, 2003 & Idem & Idem & 5 \\
\hline 13 & Partamona vicina Camargo, 1980 & Idem & Idem & 6 \\
\hline 14 & Scaura sp. group tenuis (Ducke, 1916) & Igarapé Açú, São Francisco, rio Negro & $02^{\circ} 49^{\prime} 58^{\prime \prime S}, 60^{\circ} 46^{\prime} 51^{\prime \prime} \mathrm{W}$ & 1 \\
\hline 15 & Scaura sp. group latitarsis (Friese, 1900) & Santa Isabel do rio Negro, Igarapé Dará & $0^{\circ} 25^{\prime} 04^{\prime \prime S}, 65^{\circ} 01^{\prime} 07^{\prime \prime} \mathrm{W}$ & 2 \\
\hline 16 & Scaura tenuis (Ducke, 1916) & Tabocal, rio Uneiuxi & $0^{\circ} 34^{\prime} 45^{\prime \prime} \mathrm{S}, 65^{\circ} 05^{\prime} 57^{\prime \prime} \mathrm{W}$ & 1 \\
\hline 17 & Scaura sp. group tenuis (Ducke, 1916) & Tapurucuara-Mirim & $0^{\circ} 25^{\prime} 17^{\prime \prime} \mathrm{S}, 66^{\circ} 24^{\prime} 22^{\prime \prime} \mathrm{W}$ & 6 \\
\hline 18 & Scaura tenuis (Ducke, 1916) & Idem & Idem & 4 \\
\hline 19 & Trigona branneri Cockerell, 1912 & Foz do Padauari, Rio Negro & $2^{\circ} 06^{\prime} 27^{\prime \prime} \mathrm{S}, 61^{\circ} 13^{\prime} 10^{\prime \prime} \mathrm{W}$ & 4 \\
\hline 20 & Trigona branneri Cockerell, 1912 & Idem & Idem & 4 \\
\hline 21 & Trigona cilipes (Fabricius, 1804) & Idem & Idem & 6 \\
\hline 22 & Trigona cilipes (Fabricius, 1804) & Tabocal, rio Uneiuxi & $0^{\circ} 24^{\prime} 45^{\prime \prime} \mathrm{S}, 65^{\circ} 05^{\prime} 57^{\prime \prime} \mathrm{W}$ & 6 \\
\hline 23 & Trigona dalatorreana Friese, 1900 & Idem & Idem & 2 \\
\hline 24 & Trigona sp. group recursa Smith, 1863 & Tapurucuara-Mirim & $0^{\circ} 25^{\prime} 17^{\prime \prime} \mathrm{S}, 66^{\circ} 24^{\prime} 22^{\prime \prime} \mathrm{W}$ & 6 \\
\hline 25 & Trigona sp. group recursa Smith, 1863 & Lago Caurés & $1^{\circ} 18^{\prime} 59^{\prime \prime} \mathrm{S}, 62^{\circ} 11^{\prime} 02^{\prime \prime} \mathrm{W}$ & 3 \\
\hline 26 & Trigona williana Friese, 1900 & Foz do Padauari, Rio Negro & $2^{\circ} 06^{\prime} 27^{\prime \prime} \mathrm{S}, 61^{\circ} 13^{\prime} 10^{\prime \prime} \mathrm{W}$ & 6 \\
\hline
\end{tabular}

"temporary specialization" events (concentrated collection from a specific pollen source), we established a minimum representation of $90 \%$ of a single pollen type in one sample. Data on the flowering phenophase of the species present on the pollen spectra were obtained from the information found on the labels of specimens from the INPA Herbarium.

The patterns of trophic overlap between bee species were evaluated according to the Schoener Index (1968), which is given by the following formula: $\mathrm{S}=1-0.5 \Sigma$ (Pxi - Pyi), where $\mathrm{S}=$ diet overlap; $\mathrm{Pxi}=$ proportion of food item $i$ in the diet of species $x$; Pyi $=$ proportion of food item $i$ in the diet of species $y$. This index varies from 0 to 1 . The overlap is considered to be biologically significant when $\mathrm{S}$ is equal to or greater than 0.6 (Zaret \& Rand 1971; Wallace 1981). We also calculated the niche overlap indexes for the allopatric species. While the values we obtained do not have real biological value, they indicate the potential of such species to compete in cases of co-occurrence. The data for the abundance of pollen types were used to perform a cluster analysis for pairing based on Bray Curtis Index. The resulting graph is shown in an UPGMA format.

Voucher specimens of the bees taken from the analyzed nests were deposited in the "Camargo" Collection - RPSP of the Biology Department of the Faculdade de Filosofia,
Ciências e Letras de Ribeirão Preto, Universidade de São Paulo. Pollen slides were deposited in the Palinoteca of the Instituto Nacional de Pesquisas da Amazonia (INPA). João M. F. Camargo identified the bee species according to the classification proposal of Camargo and Pedro (2008). The plant nomenclature follows Tropicos (Missouri Botanical Garden - MOBOT) and the classification proposal APGII.

\section{RESULTS}

An analysis of 104 pots of pollen led to the identification of 78 pollen types, with 47 of those being identified at species level belonging to 36 botanical families (Table II). Of the total number of recorded plants, $39.7 \%$ had a confirmed flowering phenophase during the months of June, July and August. On average, the pollen pots samples contained between one and five pollen types. The bee species with the broadest pollen spectrum were Trigona williana and Trigona cilipes (nest 22), each one with fifteen pollen types, while the narrowest pollen spectrum was recorded for Scaura sp. gr. tenuis (nest 14), with only two pollen types. The lowest average of pollen type per spectrum was recorded among Scaura species (4.4). For this genus, fewer bee species and an equally low number of pollen pots (14) were evaluated; however, when we evaluated the con- 
Table II. Plant species represented in the pollen pots from nests of the genera Partamona Schwarz, 1939, Scaura Schwarz, 1938 and Trigona Jurine, 1807, and their relative occurrence in the total samples of each bee genus analyzed and for all the samples studied (104) from the Rio Negro in Amazonas, Brazil.

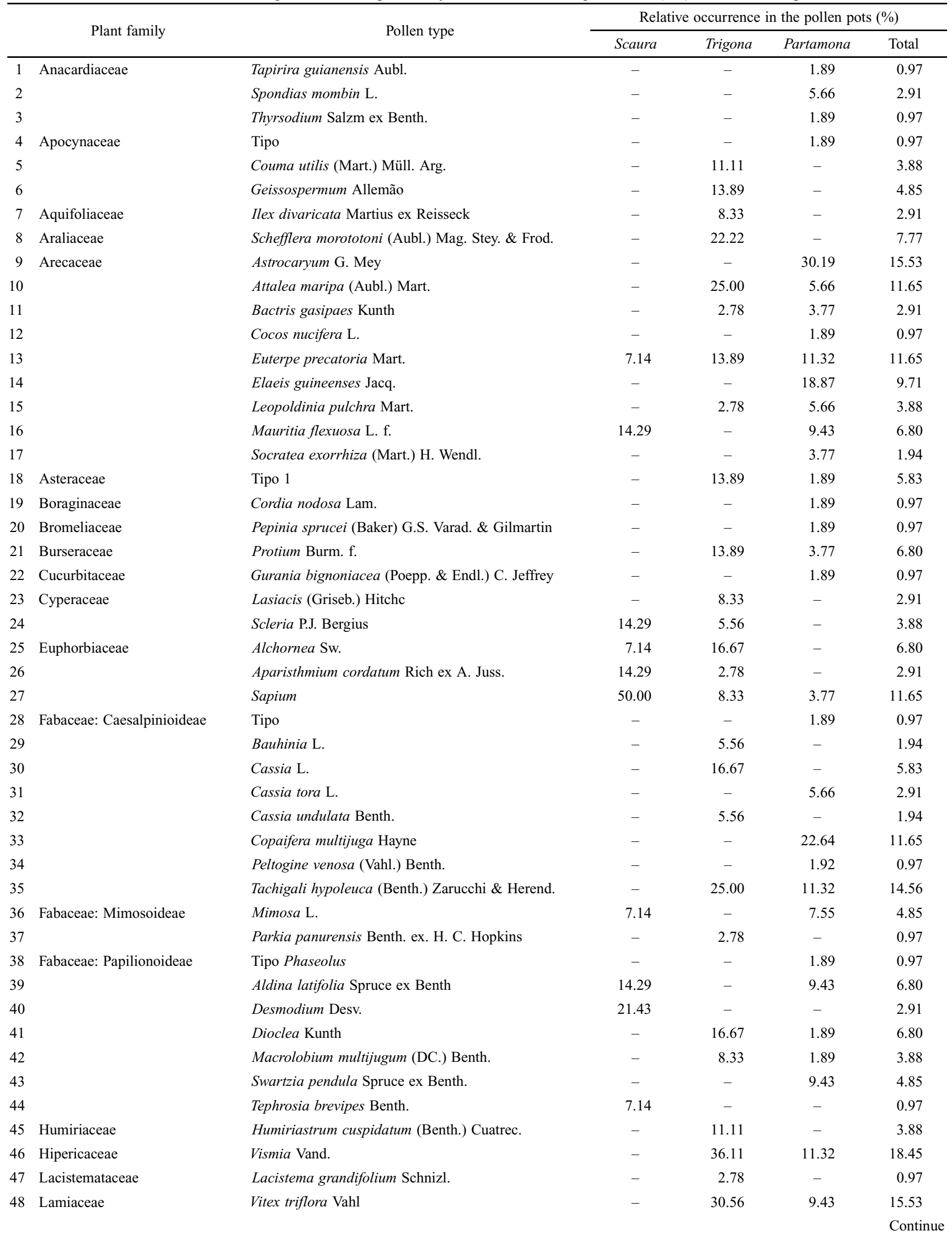


Table II. Continued.

\begin{tabular}{|c|c|c|c|c|c|c|}
\hline & \multirow{2}{*}{ Plant family } & \multirow{2}{*}{ Pollen type } & \multicolumn{4}{|c|}{ Relative occurrence in the pollen pots $(\%)$} \\
\hline & & & Scaura & Trigona & Partamona & Total \\
\hline 49 & Loranthaceae & Phthirusa rufa (Mart.) Eichler & - & 11.11 & - & 3.88 \\
\hline 50 & Malpighiaceae & Banisteriopsis C. B. Rob. & - & 2.78 & - & 0.97 \\
\hline 51 & & Burdachia prismatocarpa A. Juss. & - & 19.44 & - & 6.80 \\
\hline 52 & & Byrsonima Rich. ex Kunth & 14.29 & 25.00 & 16.98 & 19.42 \\
\hline 53 & Malvaceae: Bombacoideae & Catostemma sclerophyllum Ducke & - & - & 1.89 & 0.97 \\
\hline 54 & Malvaceae: Sterculioideae & Teobroma grandiflorum (Will. ex Spreng.) Schum. & - & 2.78 & - & 0.97 \\
\hline 55 & Melastomataceae & Bellucia imperialis Saldanha \& Cogn. & 14.29 & 16.67 & - & 7.77 \\
\hline 56 & & Miconia Ruiz \& Pav. & 35.71 & 2.78 & 18.87 & 15.53 \\
\hline 57 & Meliaceae & Carapa guianensis Aubl. & - & 5.56 & - & 1.94 \\
\hline 58 & Moraceae & Trymatococcus amazonicus Poepp. e Endl. & - & 27.78 & 1.89 & 10.68 \\
\hline 59 & Myrsinaceae & Conomorpha spicatus (Kunth) Mez & - & - & 1.89 & 0.97 \\
\hline 60 & Myrtaceae & Tipo & 7.14 & 8.33 & 50.94 & 30.10 \\
\hline 61 & Olacaceae & Aptandra liriosmoides Spruce ex Miers & - & - & 5.66 & 2.91 \\
\hline 62 & Passifloraceae & Passiflora Killip & 7.14 & - & - & 0.97 \\
\hline 63 & Piperaceae & Piper L. & - & 11.11 & - & 3.88 \\
\hline 64 & Poaceae & Tipo Pariana & 7.14 & 11.11 & - & 4.85 \\
\hline 65 & Polygalaceae & Securidaca longifolia Poepp. & - & 2.78 & 1.89 & 1.94 \\
\hline 66 & Rubiaceae & Amaioua corymbosa Kunth & - & - & 1.89 & 0.97 \\
\hline 67 & & Spermacoce L. & - & 2.78 & - & 0.98 \\
\hline 68 & & Isertia hypoleuca Benth. & - & 33.33 & - & 11.65 \\
\hline 69 & Rutaceae & Zanthoxylum rhoifolium Lam. & 7.14 & - & 5.66 & 3.88 \\
\hline 70 & Salicaceae & Casearia Jacq. & - & 2.78 & - & 0.97 \\
\hline 71 & & Laetia suaveolens (Poepp.) Benth. & - & - & 5.66 & 2.91 \\
\hline 72 & & Lindackeria paludosa (Benth.) Gilg & 7.14 & 25.00 & 1.89 & 10.68 \\
\hline 73 & & Ryania speciosa Vahl. & - & 5.56 & - & 1.94 \\
\hline 74 & Sapindaceae & Matayba Aubl. & - & - & 1.92 & 0.97 \\
\hline 75 & & Toulicia Aubl. & - & - & 3.77 & 1.94 \\
\hline 76 & Urticaceae & Cecropia Loefl. & - & 8.33 & 13.21 & 9.71 \\
\hline 77 & & Pourouma Aubl. & - & 8.33 & - & 2.91 \\
\hline 78 & Verbenaceae & Citharexylum macrophyllum Poir. & 14.29 & - & - & 1.94 \\
\hline
\end{tabular}

tent of each pollen pot, we noticed the same average amount of the other species from the other two genera (Trigona and Partamona) that were studied.

The most commonly pollen type was Myrtaceae, which was present in $30.1 \%$ of the pollen samples analyzed. The species considered attractive (frequency $>10 \%$ ) were the following: Alchornea sp., Aptandra liriosmoides, Astrocaryum sp., Attalea maripa, Bactris gasipaes, Bellucia imperialis, Burdachia prismatocarpa, Byrsonima sp., Cassia sp., Cecropia sp., Citharexylum macrophylum, Coco nucifera, Copaifera multijuga, Desmodium sp., Dioclea sp., Elaeis guineenses, Euterpe precatoria, Isertia hypoleuca, Laetia suaveolens, Lasiacis sp., Leopoldinia pulchra, Lindackeria paludosa, Mauritia flexuosa, Miconia sp., Piper sp., Pourouma sp., Ryania speciosa, Sapium sp., Schefflera morototoni, Scleria sp., Spondias mombin, Swartzia pendula, Tachigali hypoleuca, Trymatococcus amazonicus, Vismia sp., Vitex triflora and Zanthoxylum rhoifolium (Table III). Among these species, Alchornea, Astrocaryum, Ba. gasipaes, Byrsonima, Cassia, C. multijuga, E. guineenses, E. precatoria, L. corymbulosa, L. paludosa, M. flexuosa, Miconia, Pourouma, Scleria, T. hypoleuca and Z. rhoifolium triggered temporary specialization events (frequency $>90 \%$ in one given pot).

We registered 41 events of temporary specialization (frequency $>90 \%$ ); six among 37 analyzed pots of Trigona, six among 14 pots of Scaura and 29 among 53 pollen pots of Partamona. The cluster analysis indicated that the closest associations were not based on the taxonomic proximity of the bee species (Fig. 2). None species had pollen spectra from all pots grouped together. For the overlap of the pollen niche (Table IV), only one pair of allopatric species, namely Partamona pearsoni and Partamona ferreirai (nest 12), and two nests of $P$. mourei (nests 6 and 9) showed a biologically significant overlap (greater than 0,6 ). However, one species from each pair had only one pot of pollen analyzed. 
Table III. Relative frequency (per pot) of pollen types found in the pollen pots of the Meliponini nests from the Rio Negro channel in Amazonas, Brazil. Bee species (columns) are numbered according to Table I and plant species (rows) are numbered according to Table II.

\begin{tabular}{|c|c|c|c|c|c|c|c|c|c|c|c|c|c|c|c|c|c|c|c|c|c|c|c|c|c|c|}
\hline \multirow{2}{*}{ Nests } & \multicolumn{13}{|c|}{ Partamona } & \multicolumn{5}{|c|}{ Scaura } & \multicolumn{8}{|c|}{ Trigona } \\
\hline & 1 & 2 & 3 & 4 & 5 & 6 & 7 & 8 & 9 & 10 & 11 & 12 & 13 & 14 & 15 & 16 & 17 & 18 & 19 & 20 & 21 & 22 & 23 & 24 & 25 & 26 \\
\hline 1 & - & 6.7 & - & - & - & - & - & - & - & - & - & - & - & - & - & - & - & - & - & - & - & - & - & - & - & - \\
\hline 2 & - & - & - & 14 & - & - & - & - & - & - & - & - & - & - & - & - & - & - & - & - & - & - & - & - & - & - \\
\hline 3 & - & - & - & - & - & - & - & 4.2 & - & - & - & - & - & - & - & - & - & - & - & - & - & - & - & - & - & - \\
\hline 4 & 0.3 & - & - & - & - & - & - & - & - & - & - & - & - & - & - & - & - & - & - & - & - & - & - & - & - & - \\
\hline 5 & - & - & - & - & - & - & - & - & - & - & - & - & - & - & - & - & - & - & - & - & 2.1 & - & - & - & - & - \\
\hline 6 & - & - & - & - & - & - & - & - & - & - & - & - & - & - & - & - & - & - & - & - & - & - & - & - & - & 0.1 \\
\hline 7 & - & - & - & - & - & - & - & - & - & - & - & - & - & - & - & - & - & - & - & 0.3 & - & 0.7 & - & - & - & - \\
\hline 8 & - & - & - & - & - & - & - & - & - & - & - & - & - & - & - & - & - & - & - & 9.0 & - & 0.4 & - & 27 & - & - \\
\hline 9 & - & - & - & 22 & - & 93 & - & - & 84 & 46 & 25 & - & - & - & - & - & - & - & - & - & - & - & - & - & - & - \\
\hline 10 & - & - & - & 26 & 0.2 & - & - & - & - & - & - & - & - & - & - & - & - & - & - & 16 & - & - & - & - & - & 28 \\
\hline 11 & - & - & - & - & - & - & - & - & - & - & - & - & 0.4 & - & - & - & - & - & - & - & - & - & - & 17 & - & - \\
\hline 12 & 87 & - & - & - & - & - & - & - & - & - & - & - & - & - & - & - & - & - & - & - & - & - & - & - & - & - \\
\hline 13 & - & 0.7 & - & 4.9 & - & - & - & - & - & - & 17 & 0.1 & - & - & - & 4.5 & - & - & - & 1.2 & - & 2.5 & 42 & - & - & - \\
\hline 14 & - & - & - & - & - & - & - & - & - & 53 & - & - & - & - & - & - & - & - & - & - & - & - & - & - & - & - \\
\hline 15 & - & - & - & - & 31 & - & - & - & - & - & - & - & - & - & - & - & - & - & - & - & - & 0.2 & - & - & - & - \\
\hline 16 & - & - & - & - & - & - & - & 1.4 & - & - & - & 0.1 & 47 & - & - & - & - & 21 & - & - & - & - & - & - & - & - \\
\hline 17 & 0.9 & - & - & - & - & - & - & - & 0.5 & - & - & - & - & - & - & - & - & - & - & - & - & - & - & - & - & - \\
\hline 18 & - & - & - & 0.1 & - & - & - & - & - & - & - & - & - & - & - & - & - & - & - & - & - & - & - & - & - & 0.1 \\
\hline 19 & - & - & - & - & - & - & - & 0.1 & - & - & - & - & - & - & - & - & - & - & - & - & - & - & - & - & - & - \\
\hline 20 & - & - & - & - & - & - & - & 1.0 & - & - & - & - & - & - & - & - & - & - & - & - & - & - & - & - & - & - \\
\hline 21 & - & - & - & - & - & - & - & - & - & 0.1 & 1.2 & - & - & - & - & - & - & - & - & - & - & 0.1 & - & 0.1 & - & 0.1 \\
\hline 22 & - & - & - & - & - & - & - & 0.2 & - & - & - & - & - & - & - & - & - & - & - & - & - & - & - & - & - & - \\
\hline 23 & - & - & - & - & - & - & - & - & - & - & - & - & - & - & - & - & - & - & - & - & - & - & - & 16 & - & - \\
\hline 24 & - & - & - & - & - & - & - & - & - & - & - & - & - & - & - & - & 20 & - & - & - & - & - & - & - & 0.5 & - \\
\hline 25 & - & - & - & - & - & - & - & - & - & - & - & - & - & - & - & 95 & - & - & - & - & - & 45 & - & - & 0.1 & - \\
\hline 26 & - & - & - & - & - & - & - & - & - & - & - & - & - & - & - & - & 0.1 & - & - & - & - & - & - & - & 6.1 & - \\
\hline 27 & - & 73 & - & - & - & - & 3.2 & - & - & - & - & - & - & 1.0 & 35 & - & - & 47 & - & 18 & - & - & - & 15 & - & - \\
\hline 28 & - & - & - & - & - & - & - & - & 0.2 & - & - & - & - & - & - & - & - & - & - & - & - & - & - & - & - & - \\
\hline 29 & - & - & - & - & - & - & - & - & - & - & - & - & - & - & - & - & - & - & - & - & - & - & - & - & - & 0.1 \\
\hline 30 & - & - & - & - & - & - & - & - & - & - & - & - & - & - & - & - & - & - & - & 53 & - & - & - & - & - & 0.1 \\
\hline 31 & - & - & - & - & - & 0.7 & - & - & - & - & - & - & - & - & - & - & - & - & - & - & - & - & - & - & - & - \\
\hline 32 & - & - & & & - & - & & - & - & - & - & - & & - & - & - & - & - & - & - & - & 6.0 & - & - & - & - \\
\hline 33 & - & - & 33 & 0.9 & - & - & 93 & - & - & - & - & 81 & 17 & - & - & - & - & - & - & - & - & - & - & - & - & - \\
\hline 34 & - & - & - & - & - & - & 2.3 & - & - & - & - & - & - & - & - & - & - & - & - & - & - & - & - & - & - & - \\
\hline 35 & - & - & - & - & - & - & - & - & - & - & - & 0.2 & 36 & - & - & - & - & - & 43 & - & 29 & - & - & - & - & - \\
\hline 36 & - & 4.3 & - & 0.2 & 2.4 & - & - & - & - & - & - & - & - & - & - & - & 2.6 & - & - & - & - & - & - & - & - & - \\
\hline 37 & - & - & - & - & - & - & - & - & - & - & - & - & - & - & - & - & - & - & - & - & - & 2.0 & - & - & - & - \\
\hline 38 & - & - & - & - & - & - & - & - & - & - & 1.1 & - & - & - & - & - & - & - & - & - & - & - & - & - & - & - \\
\hline 39 & - & - & - & - & - & 0.1 & - & - & - & - & 8.2 & - & - & - & 1.2 & - & - & - & - & - & - & - & - & - & - & - \\
\hline 40 & - & - & - & - & - & - & - & - & - & - & - & - & - & - & - & - & - & 30 & - & - & - & - & - & - & - & - \\
\hline 41 & 5.2 & - & - & - & - & - & - & - & - & - & - & - & - & - & - & - & - & - & - & - & 20 & - & - & - & - & - \\
\hline 42 & - & - & - & - & - & - & 0.7 & - & - & - & - & - & - & - & - & - & - & - & - & - & - & 0.3 & - & - & - & - \\
\hline 43 & - & - & - & - & - & - & - & - & - & - & - & 18 & - & - & - & - & - & - & - & - & - & - & - & - & - & - \\
\hline 44 & - & - & - & - & - & - & - & - & - & - & - & - & - & - & - & - & 0.1 & - & - & - & - & - & - & - & - & - \\
\hline 45 & - & - & - & - & - & - & - & & - & - & & - & - & - & - & - & - & - & - & - & - & - & - & - & - & 0.3 \\
\hline 46 & - & 14 & - & - & - & - & - & 0.1 & - & - & 2.0 & - & - & - & - & - & - & - & - & 0.2 & 29 & - & - & - & 19 & 1.1 \\
\hline 47 & - & - & - & - & - & - & - & - & - & - & - & - & - & - & - & - & - & - & - & 1.3 & - & - & - & - & - & - \\
\hline 48 & 4.5 & - & - & - & - & - & - & 15 & - & - & 3.5 & - & - & - & - & - & - & - & 0.2 & - & - & - & - & - & - & 2.3 \\
\hline
\end{tabular}


Table III. Continued.

\begin{tabular}{|c|c|c|c|c|c|c|c|c|c|c|c|c|c|c|c|c|c|c|c|c|c|c|c|c|c|c|}
\hline \multirow{2}{*}{ Nests } & \multicolumn{13}{|c|}{ Partamona } & \multicolumn{5}{|c|}{ Scaura } & \multicolumn{8}{|c|}{ Trigona } \\
\hline & 1 & 2 & 3 & 4 & 5 & 6 & 7 & 8 & 9 & 10 & 11 & 12 & 13 & 14 & 15 & 16 & 17 & 18 & 19 & 20 & 21 & 22 & 23 & 24 & 25 & 26 \\
\hline 49 & - & - & - & - & - & - & - & - & - & - & - & - & - & - & - & - & - & - & - & - & - & 9.5 & - & - & - & - \\
\hline 50 & 2.5 & - & - & - & - & - & - & - & - & - & - & - & - & - & - & - & - & - & - & - & 0.4 & - & - & - & - & - \\
\hline 51 & - & - & - & - & - & - & - & - & - & - & - & - & - & - & - & - & - & - & 5.4 & - & 18 & - & - & - & - & - \\
\hline 52 & - & - & 67 & 19 & - & - & - & - & - & - & - & - & - & - & 0.3 & - & - & - & 2.8 & - & - & 8.6 & - & - & 4.2 & 0.4 \\
\hline 53 & - & - & - & - & - & - & - & - & - & 0.1 & - & - & - & - & - & - & - & - & - & - & - & - & - & - & - & - \\
\hline 54 & - & - & - & - & - & - & - & - & - & - & - & - & - & - & - & - & - & - & - & - & - & - & - & - & - & 0.1 \\
\hline 55 & - & & - & - & - & & - & - & - & - & - & - & - & - & 37.8 & - & - & - & - & - & - & - & - & - & - & 66 \\
\hline 56 & - & 0.7 & - & - & 7.3 & 0.2 & - & - & 0.2 & - & 18 & - & - & - & - & - & 63.1 & 1.0 & - & - & - & - & - & 0.3 & - & - \\
\hline 57 & - & - & - & - & - & - & - & - & - & - & - & - & - & - & - & - & - & - & - & - & - & - & - & - & - & 0.5 \\
\hline 58 & - & - & - & - & - & - & - & 1.1 & - & - & - & - & - & - & - & - & - & - & - & - & - & 0.2 & 0.6 & 5.4 & 18 & - \\
\hline 59 & - & - & 0.4 & - & - & & - & - & - & - & - & - & - & - & - & - & - & - & - & - & - & - & - & - & - & - \\
\hline 60 & - & - & 0.1 & 0.2 & 2.3 & 3.1 & - & 5.9 & - & - & 5.4 & 0.3 & - & - & 0.58 & - & - & - & - & - & 0.1 & - & - & - & 0.3 & - \\
\hline 61 & - & - & - & - & 3.4 & - & - & - & - & - & 11 & - & - & - & - & - & - & - & - & - & - & - & - & - & - & - \\
\hline 62 & - & - & - & - & - & - & - & - & - & - & - & - & - & - & - & - & 0.1 & - & - & - & - & - & - & - & - & - \\
\hline 63 & - & - & - & - & - & - & - & - & - & - & - & - & - & - & - & - & - & - & - & - & - & - & - & 15 & - & - \\
\hline 64 & - & - & - & - & - & - & - & - & - & - & - & - & - & - & - & 0.5 & - & - & - & - & - & - & - & 4.7 & - & - \\
\hline 65 & - & - & - & - & - & - & - & - & - & - & 0.2 & - & - & - & - & - & - & - & - & - & - & 0.4 & - & - & - & - \\
\hline 66 & - & - & - & - & - & - & - & - & - & - & - & 0.1 & - & - & - & - & - & - & - & - & - & - & - & - & - & - \\
\hline 67 & - & - & - & - & - & - & - & - & - & - & - & - & - & - & - & - & - & - & - & - & - & & - & - & - & 0.1 \\
\hline 68 & - & - & - & - & - & - & - & - & - & - & - & - & - & - & - & - & - & - & - & - & - & 16 & - & - & 3.2 & 0.5 \\
\hline 69 & - & - & - & - & - & - & - & 32 & - & - & - & - & - & - & - & - & 14 & - & - & - & - & - & - & - & - & - \\
\hline 70 & - & - & - & - & - & - & - & - & - & - & - & - & - & - & - & - & - & - & - & - & 0.9 & - & - & - & - & - \\
\hline 71 & - & - & - & - & 54 & 3.4 & - & - & - & - & - & - & - & & - & - & - & - & - & - & - & & - & - & - & - \\
\hline 72 & - & - & - & - & - & - & - & - & - & 0.2 & - & - & - & 99 & - & - & - & - & 31 & - & & 8.1 & - & - & - & - \\
\hline 73 & - & - & - & - & - & - & - & - & - & - & - & - & - & - & - & - & - & - & 18 & - & 0.2 & - & - & - & - & - \\
\hline 74 & - & - & - & - & - & - & - & 0.1 & - & - & - & - & - & - & - & - & - & - & - & - & - & - & - & - & - & - \\
\hline 75 & - & 0.8 & - & & - & - & - & - & - & - & 2.2 & - & - & - & - & - & - & - & - & - & - & - & - & - & - & - \\
\hline 76 & - & - & - & 13 & - & - & - & 39 & 15 & - & 4.4 & - & 0.1 & - & - & - & - & - & - & - & - & - & - & - & 47 & - \\
\hline 77 & - & - & - & - & - & - & - & - & - & - & - & - & - & - & - & - & - & - & - & - & - & - & 57 & - & 1.5 & - \\
\hline 78 & - & - & - & - & - & - & - & - & - & - & - & - & - & - & 25 & - & - & - & - & - & - & - & - & - & - & - \\
\hline
\end{tabular}

\section{DISCUSSION}

The clustering pattern of the nests did not reveal any pollen collection pattern that was characteristically inter- or intra-specific. These findings are in contrast to much of the previous literature regarding utilization of pollen resources by bees, in which the phylogeny (degree of relationship among taxa) and especially the phylogenetic/location association of nests were found to be the main determinants in the composition of the pollen spectra (Sommeijer et al. 1983; Biesmeijer \& van Nieuwstadt 1997; Nagamitsu et al. 1999; Biesmeijer and Slaa 2004). In a study of three Trigona species (sensu stricto), for instance, was found a greater overlap in intraspecific pollen niches than among species of the same genus (Eltz et al. 2001).

Considering that the analysis presented here is based on naturally occurring nests, we believe that an explanation for the clustering pattern we found could be related to the fol- lowing two factors: the location of the nests and the sharing of resources. Because the frequency of Meliponini nests inside the forest is usually low (Michener 1946; Hubbell \& Johnson 1977; Oliveira et al. 1995), we hypothesize that some nests may be just as isolated from the others in the same sampling area as from others located in different sampling areas. In this way, the succession of massive and rapid flowering events at the various locations where the bees are present would explain the existence of pots of distinct nests being grouped in one set and pots from the same nests not being grouped (Fig. 2).

The second factor relates to nests located in such a way that there would be an overlap in the different bees' collection areas. In this case, there is the possibility of shared resources among the different species of bees, which would make it possible to find some species of plants being shared among nests in the same sampling point regardless of the bee species, while the main pollen type in the spectra of each 
Table IV. Index of the overlap of the pollen trophic niche between pairs of bee nests. Column and row numbers indicate bee nests as listed in Table I.

\begin{tabular}{|c|c|c|c|c|c|c|c|c|c|c|c|c|c|c|c|c|c|c|c|c|c|c|c|c|c|}
\hline N. & 1 & 2 & 3 & 4 & 5 & 6 & 7 & 8 & 9 & 10 & 11 & 12 & 13 & 14 & 15 & 16 & 17 & 18 & 19 & 20 & 21 & 22 & 23 & 24 & 25 \\
\hline 1 & 1 & & & & & & & & & & & & & & & & & & & & & & & & \\
\hline 2 & - & 1 & & & & & & & & & & & & & & & & & & & & & & & \\
\hline 3 & 0.03 & - & 1 & & & & & & & & & & & & & & & & & & & & & & \\
\hline 4 & 0.02 & 0.01 & 0.20 & 1 & & & & & & & & & & & & & & & & & & & & & \\
\hline 5 & - & 0.03 & $*$ & 0.01 & 1 & & & & & & & & & & & & & & & & & & & & \\
\hline 6 & - & $*$ & $*$ & 0.23 & 0.03 & 1 & & & & & & & & & & & & & & & & & & & \\
\hline 7 & - & 0.03 & 0.33 & 0.01 & - & - & 1 & & & & & & & & & & & & & & & & & & \\
\hline 8 & 0.05 & $*$ & $*$ & 0.13 & 0.02 & 0.03 & - & 1 & & & & & & & & & & & & & & & & & \\
\hline 9 & 0.01 & $*$ & - & 0.35 & $*$ & 0.85 & - & 0.15 & 1 & & & & & & & & & & & & & & & & \\
\hline 10 & - & - & - & 0.22 & - & 0.46 & - & - & 0.46 & 1 & & & & & & & & & & & & & & & \\
\hline 11 & 0.04 & 0.04 & $*$ & 0.31 & 0.13 & 0.26 & - & 0.13 & 0.30 & 0.25 & 1 & & & & & & & & & & & & & & \\
\hline 12 & - & $*$ & 0.33 & 0.01 & $*$ & $*$ & 0.81 & $*$ & - & - & * & 1 & & & & & & & & & & & & & \\
\hline 13 & - & - & 0.17 & 0.01 & - & - & 0.17 & $*$ & $*$ & - & $*$ & 0.17 & 1 & & & & & & & & & & & & \\
\hline 14 & - & 0.01 & - & - & - & - & 0.01 & - & - & - & - & - & - & 1 & & & & & & & & & & & \\
\hline 15 & $*$ & 0.35 & $*$ & 0.01 & 0.01 & 0.01 & 0.03 & 0.01 & - & - & 0.02 & $*$ & - & 0.01 & 1 & & & & & & & & & & \\
\hline 16 & - & 0.01 & - & 0.05 & - & - & - & - & - & - & 0.05 & $*$ & - & - & - & 1 & & & & & & & & & \\
\hline 17 & - & 0.03 & - & $*$ & 0.10 & $*$ & - & 0.14 & $*$ & - & 0.18 & - & - & - & - & - & 1 & & & & & & & & \\
\hline 18 & - & 0.48 & - & - & 0.01 & $*$ & 0.03 & 0.01 & $*$ & - & 0.01 & $*$ & 0.21 & 0.01 & 0.35 & - & 0.01 & 1 & & & & & & & \\
\hline 19 & 0.03 & - & 0.03 & 0.03 & - & - & - & $*$ & - & $*$ & $*$ & $*$ & 0.36 & 0.31 & $*$ & - & - & - & 1 & & & & & & \\
\hline 20 & - & 0.19 & - & 0.17 & $*$ & - & 0.03 & $*$ & - & - & 0.01 & $*$ & - & 0.01 & 0.19 & - & - & 0.19 & - & 1 & & & & & \\
\hline 21 & 0.05 & 0.14 & $*$ & $*$ & $*$ & $*$ & - & $*$ & - & - & 0.02 & $*$ & 0.29 & - & $*$ & - & - & - & 0.35 & $*$ & 1 & & & & \\
\hline 22 & 0.11 & 0.01 & 0.07 & 0.09 & $*$ & - & 0.01 & $*$ & - & $*$ & 0.02 & $*$ & - & 0.07 & 0.10 & 0.39 & - & - & 0.10 & 0.02 & - & 1 & & & \\
\hline 23 & - & 0.01 & - & 0.05 & - & - & - & 0.01 & - & - & 0.17 & $*$ & - & - & - & 0.05 & - & - & - & 0.01 & - & 0.02 & 1 & & \\
\hline 24 & - & 0.15 & - & - & $*$ & $*$ & 0.03 & 0.01 & $*$ & $*$ & $*$ & - & $*$ & 0.15 & 0.15 & 0.01 & $*$ & 0.15 & - & 0.24 & - & 0.01 & 0.01 & 1 & \\
\hline 25 & 0.03 & 0.14 & 0.04 & 0.18 & $*$ & $*$ & - & 0.42 & 0.15 & - & 0.07 & $*$ & $*$ & - & $*$ & - & $*$ & - & 0.03 & $*$ & 0.19 & 0.12 & $*$ & 0.05 & 1 \\
\hline 26 & 0.05 & 0.05 & $*$ & 0.26 & $*$ & - & - & 0.02 & - & $*$ & 0.03 & - & - & - & 0.38 & - & - & - & $*$ & 0.16 & 0.09 & 0.09 & - & $*$ & 0.02 \\
\hline
\end{tabular}

* Overlap lower than 0.01 .

bee species would be different (Ramalho et al. 2007). This pattern was clearly observed among the Trigona species from the Foz do Padauari sampling point (nests 19, 20, 21 and 26), among which some pollen types were shared with antagonic abundances (Table III). Another example was found at the Tapurucuara-mirim sampling point between the species $P$. ferreirai and Partamona vicina. P. ferreirai focused its foraging activity on Copaifera multijuga, with the pollen spectrum of all pots showing the prevalence of this plant, apart from one single pot. On the other hand, for $P$. vicina we observed the prevalence of the same plant, C. multijuga, in only one pot, suggesting that the both species could have succeeded in collecting this resource.

Sharing of pollen resources among different species of bee has been reported in several studies on Atlantic vegetation. However, it is rare to find the same flower as the major source of pollen for the different species of bees being compared (Ramalho et al. 2007). A vast niche overlap was reported for the pollen sources of Meliponini and Apis mellifera L. 1758 in the studies performed in the Atlantic forest. Such overlap was attributed to very productive flowering, where the chances of competition are lower (Ramalho 1990; Ramalho et al. 1991, 2007). Given the speculative nature of the explanations of- fered here, we suggest that additional systematic research is needed to address the issue of the sharing of pollen resources in the natural environment of the Amazon region.

The biologically significant overlap in the pollen niches reported here have no real biological value because the bee species with overlapping niches did not occur in the same locations. However, it may be important to consider the potential characteristics of the plants involved in the overlap (in this particular case, Astrocaryum sp. and C. multijuga) for beekeeping initiatives and to fully realize the importance of these plant species for bees. It is also noteworthy that the four species whose niches overlapped belong to the genus Partamona; the nests of one pair were found in Igapó vegetation (wetland) and the other in Terra firme vegetation (land).

The data obtained here for Trigona williana confirm those from Marques-Souza et al. (1996), who, in their work with the same species, reported abundant pollen collection in species from the genera Attalea and Bellucia. Besides these two species, the authors identified 56 more pollen types collected by $T$. williana over a 1-yr period. They observed that the lowest total number of pollen types was collected during the rainy season. According to the authors, the competition for food intensifies during that season as the supply diminishes and, 


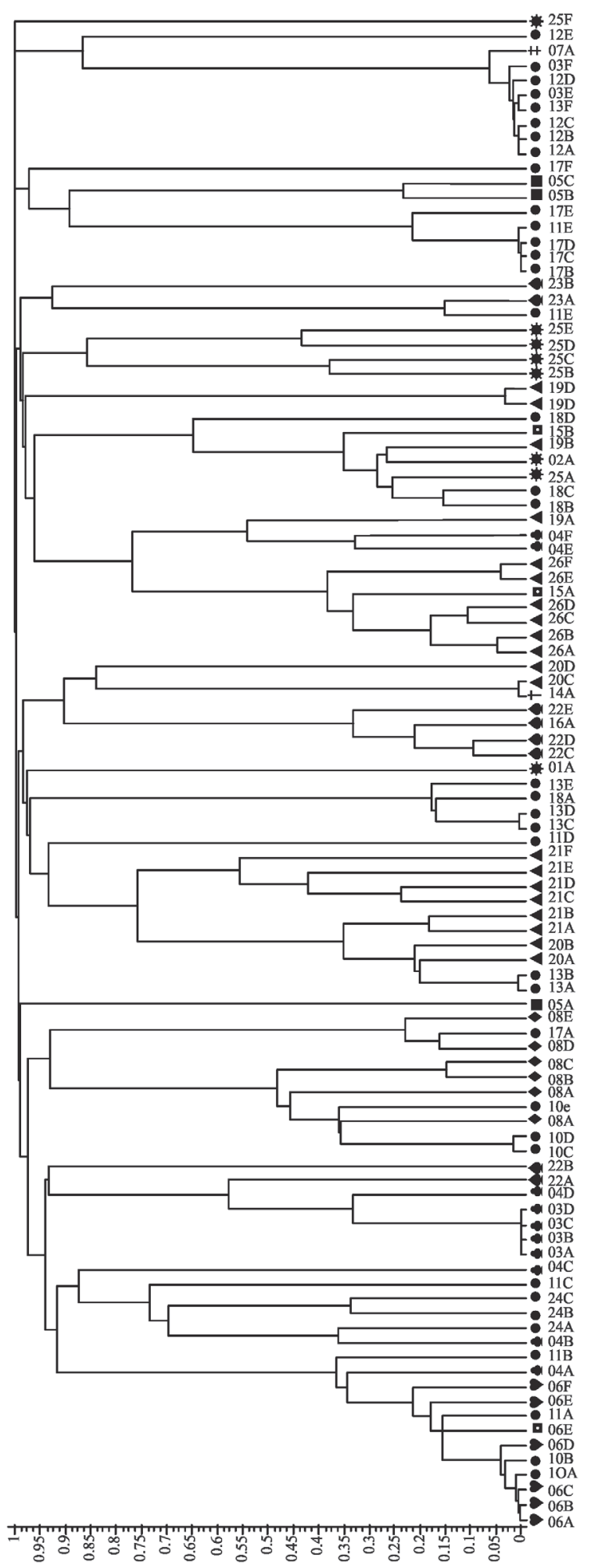

Fig. 2. Clustering of the Meliponini nests of Partamona, Scaura and Trigona according to the identity of the pollen spectra using the "pairing method" as a function of the Bray Curtis Index. Bee nests are numbered according to Table I and letters represent the different pots analyzed from the same nest. Each symbol represent different places as follow: Tapurucuara-Mirim, - Ponta Camucuri, Tabocal-Rio Uneiuxi, \& Nazaré do Rio Negro, - Sta. Isabel do Rio Negro-Igarapé do Dará, ‡ Samauma-Rio Dará, $\boldsymbol{\Delta}$ Foz do Padauari, $\bullet$ Rio Demeni-"Pai Raimundo", * Lago Caurés, and † Igarapé Açu-São Francisco, $\bullet$ Santa Helena, Ponta do Gavião, Rio Negro. in this case, the strategy of mass collection associated with aggression may lead to an increase in the pollen spectrum. In the present study, however, the amplitude of the pollen spectrum per pot of pollen analyzed was not greater for Trigona than for any other genera analyzed.

Although the pollen grain type Myrtaceae was found in $30.1 \%$ of the pollen pots, its frequency was not sufficiently high for it to be considered attractive. We believe that this fact may be related to the onset of the flowering phenophase of some widely distributed Myrtaceae species, because pollen from plants of this family is usually intensively collected when available (Absy et al. 1984; Marques-Souza et al. 1993, 2007; Ramalho et al. 1989, 2007). Moreover, there are many reports of the pollination of Myrtaceae being attributed to the Meliponini (Gressler et al. 2006; Cortopassi-Laurino \& Ramalho 1988).

Two types of pollen from Cyperaceae (Scleria and Lasiacis), collected only by T. recursa, Trigona sp. gr. recursa and Scaura tenuis, were considered attractive. This is particularly interesting because the Cyperaceae family does not have flowers that are considered attractive to bees and its pollen is light and dry, consistent with anemophily pollination (Vogel 1954; Faegri \& Pijl 1979; Fenster et al. 2004). One possible explanation for this finding may be pollen shortage, a phenomenon previously described in Meliponini by others (Ducke 1902; Adams et al. 1981; Terrell \& Batra 1984). Alternatively, there may be floral traits that attract visitors to the flowers; studies on pollen morphology of the species Pariana (Poaceae) and the presence of insects in the flowers suggest possible involvement of insects in its pollination mechanisms (Salgado-Labouriau et al. 1993). The authors hypothesize that the graminoids in the understory of tropical forest may require the involvement of insects for pollination when anemophily is compromised by moisture.

Along the same lines, other pollen types from anemophilous plants have been recorded (Alchornea, Cecropia, Pouroma and Piper). Just as in Cyperaceae, these plants do not possess features obviously attractive to bees (Vogel 1954; Faegri \& Pijl 1979). However, all the genera mentioned produce copious amounts of pollen and this might be a very important feature for bees that continuously need pollen in large quantities to replenish brood cells (Michener 2000). Furthermore, collection of anemophilous pollen may be further intensified in periods of pollen shortage; in the Amazon, this corresponds to the rainy season when there is a reduced number of species in the flowering phenophase, which makes pollen less available as a function of the high humidity and constant rainfall (Schaik et al. 1993; Bentos et al. 2008).

The species of Arecaceae corroborate the hypothesis of attractiveness due to resource abundance. In our study, only C. nucifera, Euterpe precatoria and Leopoldinia pulchra belong to the group of palm trees primarily pollinated by bees and, secondarily, by beetles (Listabarth 1996; Henderson et al. 2000). The other genera, namely Astrocaryum, Bactris, Attalea, Mauritia and Elaeis, belong to the cantharophilic group, in which bees are secondary pollinators (Storti 1993; Henderson et al. 2000; Tandon et al. 2001). Regardless of 
the effectiveness of bees as pollen vectors for palm trees, the abundance and constant supply of pollen throughout the year seems to make Arecaceae an especially important resource for Meliponini (Henderson 1986; Tomlinson 1990; Storti 1993; Henderson et al. 2000). In a study of T. williana, eleven species of palm trees were recorded, with of the majority of them making pollen available for more than two months, including some species such as $A$. maripa and $C$. nucifera that were recorded in the pollen spectra for more than ten months (Marques-Souza et al. 1996).

Although not as prolific in pollen production as the previously mentioned species, C. macrophylum and Isertia hypoleuca were considered attractive for the Scaura and Trigona species, respectively. The floral morphology of cogeneric species Citharexylum myrianthum Cham. is compatible with the phalenophily syndrome. Previous studies have reported moth visits and their effectiveness in this mechanism of pollination and these studies indicated that Meliponini acted as pollen robbers (Rocca \& Sazima 2006). Likewise, the morphology of I. hypoleuca is consistent with ornithophily pollination syndrome, in that visits by hummingbirds have been recorded at an appropriate frequency to make them potential pollinators (Giacomini \& Webber, unpublished data).

Among the species whose morphology is consistent with melittophily syndrome, many are not effectively pollinated by the Meliponini we studied here. This is mainly due to the small size of these bees, which does not allow for legitimate visits, and also to the destructive and robber habits of some species, especially of Partamona and Trigona. Among the pollen types of melittophilous plants that are not pollinated by Meliponini are those from species with poricidal anthers such as Cassia spp., Miconia sp. and B. imperialis, which require floral visitors capable of vibrating the anthers to remove the pollen ("buzz pollination") (Buchmann 1983; Renner 1983). Because the bees we studied are non-vibrating, it appears that Trigona spp. damage the anthers with their mandibles and then rob the pollen (Renner 1983), while the remaining bees either use the hole produced by Trigona spp. to collect pollen or act as "garbage collectors" by removing the pollen remaining after the collection by vibrating bees (Wille 1963).

Another group of melittophilous plants not pollinated by the bees we studied here includes Malpighiaceae (Byrsonima and B. prismatocarpa), two species of Fabaceae ( $S$. pendula and Dioclea sp.) and one species of Salicaceae (R. speciosa), which are only pollinated by larger solitary bees. The Malpighiaceae flowers have oil glands in their sepals and are mainly pollinated by solitary bees (Centridini, Tapinotaspidini and Tetrapediini tribes) specialized in collecting oils, that they use as larval food and nest-construction material (Teixeira \& Machado 2000; Machado 2004; Rêgo et al. 2006). The Fabaceae ( $S$. pendula and Dioclea sp.) require floral visitors larger than the Meliponini for contact to occur with the fertile parts of the flower (Lopes \& Machado 1996; Pinheiro \& Sazima 2007; Dutra et al. 2009), in the same way that $R$. speciosa is only pollinated by Euglossini bees (Martiniano, unpublished data).
C. multijuga (Freitas \& Oliveira 2002), Vismia sp. (Santos \& Machado 1998), S. mombin (Nadia et al. 2007), Z. rhoifolium (Bawa 1985b), L. paludosa (Flores \& Webber, unpublished data), Desmodium sp. (Willmer et al. 2009) and T. hypoleuca (Venturieri 1997) are among the species visited and potentially pollinated by the Meliponini we studied here. In addition to these species, the Meliponini are thought to influence the pollination mechanisms of Vitex spp., L. suaveolens, $S$. morototoni and Sapium spp. because, although no specific data is available in the literature, these species have a floral morphology consistent with pollination by insects, and they are frequently found in the pollen spectra of Meliponini from the Amazon (Absy et al. 1980, 1984; Engel \& Dingemans-Bakels 1980; Marques-Souza et al. 1995, 1996).

Based on the results presented in this study and the studies by Absy et al. (1984) and Rech \& Absy (in press.), and considering the large number of plants identified as pollen sources for bees, it is evident that further analyses are needed to verify the real need for artificial feeding of native bees in beekeeping in the Amazon. Although not all plants have a flowering phenophase that coincides with the rainy season, we show here that with proper planning, it may possible to define and cultivate plants that allow for the existence of pollen calendars convenient to the reality of each beekeeper. The species of Copaifera, Bactris, Bellucia, Byrsonima, Astrocaryum, Euterpe, Mauritia, Pouroma and Spondias may be especially interesting given the availability of pollen for bees and the possibility that they may be utilized further by human and animal populations for their fruits, seeds and oil. Moreover, species that usually grow spontaneously and are selectively removed by farmers, such as Cecropia, Sapium, Schefflera and Vismia, may also serve as pollen sources for bees if they are not eliminated.

\section{ACKNOWLEDGEMENTS}

This work is dedicated to the memory of Dr. João Maria Franco Camargo, in recognition of the brilliance of his career and the significant contributions he made in advancing our understanding of the biology of stingless bees. We thank Silvia Pedro and Caroline Scherer for reading this manuscript and making valuable suggestions, and Cleonice de Moura Oliveira, Ana Paula Moraes and Mauricio Fernandez Otarola for general helping. This work was financially supported by grants from CNPq, Processes 472563/2006-8 and 472246/ 2008-9, a scholarship to A. Rech, the primary author, and FAPESP process 1998/11605-9 that financially supported the Rio Negro collection expedition.

\section{REFERENCES}

Absy, M. L. \& W. E. Kerr. 1977. Algumas plantas visitadas para obtenção de pólen por operárias de Melipona seminigra merrilae em Manaus. Acta Amazonica 7: 309-315.

Absy, M. L.; E. B. Bezerra \& W. E. Kerr. 1980. Plantas nectaríferas utilizadas por duas espécies de Melipona da Amazônia. Acta Amazonica 10: 271-281. 
Absy, M. L.; J. M. F. Camargo; W. E. Kerr \& I. P. A. Miranda. 1984. Espécies de plantas visitadas por Meliponinae (Hymenoptera; Apoidea), para coleta de pólen na região do médio Amazonas. Revista Brasileira de Biologia 44: 227-37.

Adams, D. E.; E. Perkins \& J. R. Estes. 1981. Pollination system in Paspalum dilatatum Poir (Poaceae): na example of insect pollination in temperate grass. American Journal of Botany 68: 389-394.

Bawa, K. S. 1990. Plant-Pollinator Interactions in Tropical Rain Forests. Annual Review of Ecology and Systematics 21: 399-422.

Bawa, K. S.; D. R. Perry \& J. H. Beach. 1985a. Reproductive biology of tropical lowland rain forest trees. I. Pollination Systems and selfincompatibility mechanisms. American Journal of Botany 72: 331345.

Bawa, K. S.; D. R. Perry; S. H. Bullock; R. E. Coville \& M. H. Grayum. 1985b. Reproductive biology of tropical lowland rain forest trees. II. Pollinations mechanisms. American Journal of Botany 72: 346-356.

Bentos, T. V.; R. C. G. Mesquita \& G. B. Williamson. 2008. Reproductive phenology of central amazon pioneer trees. Tropical Conservation Science 1: 186-203.

Biesmeijer, J. C. \& E. J. Slaa. 2004. Information flow and organization of stingless bee foraging. Apidologie 35: 143-157.

Biesmeijer, J. C. \& M. G. L. van Nieuwstadt. 1997. Colony foraging patterns of stingless bees in Costa Rica, with special reference to pollen, p. 3557. $I n$ : The organization of foraging in stingless bees of the genus Melipona. Netherlands, Utrecht University, 261 p.

Biesmeijer, J. C.; E. J. Slaa; M. S. Castro; B. F. Viana; A. M. P Kleinert \& V. L. Imperatriz-Fonseca. 2005. Connectance of brazilian social bee food plant networks is influenced by habitat, but not by latitude, altitude or network size. Biota Neotropica 5: 1-8.

Buchmann, S. L. 1983. Buzz Pollination in Angiosperms. p. 73-114. In: Jones, C. E. \& Little, R. J. (eds.) Handbook of experimental pollination biology. New York, Reinhold Company, $558 \mathrm{p}$

Camargo, J. M. F. \& S. R. M. Pedro. 2008. Meliponini Lepeletier, 1836. In: Moure, J. S., Urban, D.; Melo, G. A. R. (Orgs). Catalogue of Bees (Hymenoptera, Apoidea) in the Neotropical Region - online version. Available from http://www.moure.cria.org.br/catalogue (accessed 13 August 2008).

Cortopassi-Laurino, M. \& M. Ramalho. 1988. Pollen harvest by africanized Apis mellifera and Trigona spinipes in São Paulo. Botanicals and ecological views. Apidologie 19: 01-24.

Ducke, A. 1902. Beobachtungen über blütenbesuch, erscheinungszeit etc. der bei Pará vorkommenden Bienen. II. Allgemeine Zoologie Entomologie 7: 321-326 [325]

Dutra, V. F.; M. F. Vieira; F. C. P. Garcia \& H. C. Lima. 2009. Fenologia reprodutiva, síndromes de polinização e dispersão em espécies de Leguminosae dos Campos Rupestres do Parque Estadual do Itacolomi, Minas Gerais, Brasil. Rodriguésia 60: 371-387.

Ebeling, A.; A. M. Klein; J. Schumacher; W. W. Weisser \& T. Tscharntke. 2008. How does plant richness affect pollinator richness and temporal stability of flower visits? Oikos 117: 1808-1815.

Eltz, T.; C. A. Bruhl; S. van der Kaars; V. K. Chey; K. E. Linsenmair. 2001. Pollen foraging and resource partitioning of stingless bees in relation to flowering dynamics in a Southeast Asian tropical rainforest. Insectes Sociaux 48: 273-279.

Engel, M. S. \& F. Dingemans-Bakels. 1980. Nectar and pollen resources for stingless bees (Meliponinae, Hymenoptera) in Surinam (South America). Apidologie 11: 341-350.

Ennos, R. A. 1994. Estimating the relative rates of pollen and seed migration among plant populations. Heredity 72: $250-259$.

Erdtman, G. 1960. The acetolysis method. A revided description. Svensk Botanisk Tidskrift 54: 561-564.

Faegri K. \& L. van der Pijl. 1979. The principles of pollination ecology. Oxford, Pergamon Press, 244 p.

Fenster, C. B.; W. S. Armbruster; P. Wilson; M. R. Dudash \& J. D. Thomson. 2004. Pollination syndromes and floral specialization. Annual Review of Ecology Evolution and Systematics 35: 375-403.

Freitas, C. V. \& P. E. Oliveira. 2002. Biologia reprodutiva de Copaifera langsdorffii Desf. (Leguminosae, Caesalpinioideae). Revista Brasileira de Botânica 25: 311-321.

Gressler, E.; M. A. Pizo \& L. P. C. Morellato. 2006.Polinização e dispersão de sementes em Myrtaceae do Brasil. Revista Brasileira de Botânica 29: $509-530$.

Henderson, A. 1986. A Review of pollination studies in the Palmae. The Botanical Review 52: 222-255.

Henderson, A.; B. Fischer; A. Scariot; M. W. Pacheco \& A. Pardini. 2000 Flowering phenology of a palm community in a central Amazon forest. Brittonia 52: 149-159.

Hubbell S. P. \& L. K. Johnson. 1977. Competition and nest spacing in a tropical stingless bee community. Ecology 58: 949-963.

Imperatriz-Fonseca, V. L.; M. Ramalho \& A. Kleinert-Giovannini. 1993. Flores e Abelhas de São Paulo. São Paulo, Edusp/FAPESP, 192 p.

Kerr, W. E.; M. L. Absy \& A. C. M. Souza. 1986. Espécies nectaríferas e poliníferas utilizadas pela abelha Melipona compressipes fasciculata (Meliponinae-Apidae) no Maranhão. Acta Amazonica 16-17:145-156.

Kevan, P. \& V. L. Imperatriz-Fonseca. 2002. Pollinating bees: a conservation link between agriculture and nature. Brasilia, Ministério do Meio Ambiente, $313 \mathrm{p}$.

Listabarth, C. 1996. Pollination of Bactris by Phyllotrox and Epurea. Implications of the palm breeding beetles on pollination at the community level. Biotropica 28: 69-81.

Lopes, A. V. F. E. \& I. C. S. Machado. 1996. Biologia floral de Swartzia pickelli (Leguminosae-Papilionoideae) e sua polinização por Eulaema spp. (Apidae-Euglossinae). Revista Brasileira de Botânica 19: 17-24.

Machado, I. C. 2004. Oil-collecting bees and related plants: a review of the studies in the last twenty years and case histories of plants occurring in NE Brazil, p. 255-281. In: Freitas, B. M. \& J. O. P. Pereira (eds.). Solitary bees conservation, rearing and management for pollination. Fortaleza, Imprensa Universitária, $285 \mathrm{p}$.

Marques-Souza, A. C. 2010. Ocorrência do pólen de Podocarpus sp. (Podocarpaceae) nas coletas de Frieseomelitta varia Lepeletier 1836 (Apidae: Meliponinae) em uma área urbana de Manaus, AM, Brasil. Acta Botanica Brasilica 24: 558-566.

Marques-Souza, A. C.; M. L. Absy; P. A. A. Condé \& H. A. Coelho. 1993. Dados da obtenção de pólen por operárias de Apis mellifera no município de Ji-Paraná (RO), Brasil. Acta Amazonica 23: 59-76.

Marques-Souza, A. C.; M. L. Absy; W. E. Kerr \& F. J. Aguilera-Peralta. 1995. Pólen coletado por duas espécies de meliponíneos (Hymenoptera: Apidae) da Amazônia. Revista Brasileira de Biologia 55: 855-864.

Marques-Souza, A. C.; M. L. Absy \& W. E. Keer. 2007. Pollen harvest features of the Central Amazonian bee Scaptotrigona fuvicutis Moure 1964 (Apidae: Meliponinae), in Brazil. Acta Botanica Brasilica 21: 11-20.

Marques-Souza, A. C.; I. P. A. Miranda; C. O. Moura; A. Rabelo \& E. M. Barbosa. 2002. Características morfológicas e bioquímicas do pólen coletado por cinco espécies de meliponíneos da Amazônia Central. Acta Amazonica 32: 217-229.

Marques-Souza, A. C.; C. O. Moura \& B. W. Nelson. 1996. Pollen colected by Trigona williana Hymenoptera: Apidae) in Central Amazonia. Revista de Biologia Tropical 44: 567-573.

Michener, C. D. 1946. Notes on the habits of some Panamanian stingless bees (Hymenoptera, Apidae). Journal of the New York Entomology Society 54: 179-197.

Michener, C. D. 2000. The Bees of the World. Baltimore, John Hopkins University Press, $877 \mathrm{p}$.

Nadia, T. L.; I. C. Machado \& A. V. Lopes. 2007. Polinização de Spondias tuberosa Arruda (Anacardiaceae) e análise da partilha de polinizadores com Ziziphus joazeiro Mart. (Rhamnaceae), espécies frutíferas e endêmicas da caatinga. Revista Brasileira de Botânica 30: 89-100.

Nagamitsu, T.; K. Momose; T. Inoue \& D. W. Roubik. 1999. Preference in flower visits and partitioning in pollen diets of stingless bees in an Asian tropical rain forest. Researches on Population Ecology 41: 195-202.

Oliveira, M. L.; Morato, E. F. \& Garcia, M. V. B. 1995. Diversidade de espécies e densidade de ninhos de abelhas sociais sem ferrão (Hymenoptera, Apidae, Meliponinae) em florestas de terra firme na Amazônia Central. Revista Brasileira de Zoologia 12: 13-24. 
Pedro, S. R. M. \& J. M. F. Camargo. 1991. Interactions on floral resources between the Africanized honey bee Apis mellifera L. and the native bee community (Hymenoptera: Apoidea) in a natural "cerrado" ecosystem in southeast Brazil. Apidologie 22: 397-415.

Pinheiro, M. T. \& M. Sazima. 2007. Visitantes florais e polinizadores de seis espécies arbóreas de Leguminosae melitófilas na Mata Atlântica no sudeste do Brasil. Revista Brasileira de Biociências 5: 447-449.

Ramalho, M. 1990. Foraging by stingless bees of the genus, Scaptotrigona (Apidae, Meliponinae). Journal of Apicultural Research 29: 61-67.

Ramalho, M.; L. S. Guibu; T. C. Giannini \& A. Kleinert-Giovannini. 1991. Ecologia nutricional de abelhas sociais, p. 225-252 In: Panizzi, A. R. \& J. R. P. Parra (eds.) Ecologia nutricional de insetos e suas implicações no manejo de pragas. São Paulo, Ed. Manole Ltda. 359 p.

Ramalho, M.; A. Kleinert-Giovannini \& V. L. Imperatriz-Fonseca. 1989. Utilization of floral resources by species of Melipona (Apidae: Meliponina): floral preferences. Apidologie 20: 185-195.

Ramalho, M.; M. D. Silva \& C. A. L. Carvalho. 2007. Dinâmica de uso de fontes de pólen por Melipona scutellaris Latreille (Hymenoptera: Apidae): uma análise comparativa com Apis mellifera L. (Hymenoptera: Apidae), no Domínio Tropical Atlântico. Neotropical Entomology 36: 38-45.

Ramalho, M; V. L. Imperatriz-Fonseca \& A.Kleinert-Giovannini. 1985. Exploitation of floral resources by Plebeia remota Holmberg (ApidaeMeliponinae). Apidologie 16: 307-330.

Rêgo, M. M. C.; P. M. C. Albuquerque; M. C. Ramos \& L. M. Carreira. 2006. Aspectos da biologia de nidificação de Centris flavifrons (Friese) (Hymenoptera: Apidae, Centridini), um dos principais polinizadores do murici (Byrsonima crassifolia (L.) Kunth, Malpighiaceae), no Maranhão. Neotropical Entomology 35: 579-587.

Renner, S. 1983. The widespread occurrence of anther destruction by Trigona bees in Melastomataceae. Biotropica 15: 251-256.

Rocca, M. A. \& M. Sazima. 2006. The dioecious, sphingophilous species Citharexylum myrianthum (Verbenaceae): Pollination and visitor diversity. Flora 201: 440-450.

Salgado-Labouriau, M. L.; S. Nilsson \& M. Rinaldi. 1993. Exine sculpture in Pariana pollen (Gramineae). Grana 32: 243-249.

Santos, M. J. L. \& I. C. Machado. 1998. Biologia floral e heterostilia em Vismia guianensis (Aubl.) Choisy (Clusiaceae). Acta Botanica Brasilica 12: 451-464.

Schaik, C. P.; J. W. Terborgh \& S. J. Wright. 1993. The phenology of tropical forests: adaptative significance and consequences for primary consumers. Annual Review of Ecology and Systematics 24: 353-377.

Schoener, T. W. 1968. The Anolis lizards of Bimini: resource partitioning in a complex fauna. Ecology 49: 704-726.

Simpson, B. B. \& J. L. Neff. 1981. Floral rewards: alternatives to pollen and nectar. Annals of the Missouri Botanical Garden 68: 301-322.

Sommeijer, M. J.; G. A. Rooy; W. Punt \& L. L. M. Bruijn. 1983. A comparative study of foraging behavior and pollen resources of various stingless bee (Hym., Meliponinae) and honeybees (Hym., Apinae) in Trindad, West-Indies. Apidologie 14: 205-224.

Storti, E. F. 1993. Biologia floral de Mauritia flexuosa Lin. fil, na região de Manaus, AM, Brasil. Acta Amazonica 13: 371-381.

Tandon, R.; T. N.Manohara; B. H. M. Nijalingappa \& K. R. Shivanna. 2001. Pollination and pollen-pistil interaction in oil palm, Elaeis guineensis. Annals of Botany 87: 831-838.

Teixeira, L. A. G. \& I. C. Machado. 2000. Sistema de polinização e reprodução de Byrsonima sericea DC (Malpighiaceae). Acta Botanica Brasilica 14: 347-357.

Terrell, E. E. \& S. W. T. Batra. 1984. Insect collect pollen of eastern wildrice Zizania aquatic (Poaceae). Castanea 49: 31-34.

Thiele, R. \& B. D. Inouye 2007. Nesting Biology, Seasonality, and Mating Behavior of Epicharis metatarsalis (Hymenoptera: Apidae) in Northeastern Costa Rica. Annals of the Entomological Society of America 100: 596-602.

Tomlinson P. B. 1990. The structural biology of palms. Oxford, Claredon Press Oxford, $463 \mathrm{p}$.

Venturieri, G. C. 1997. Reproductive ecology of Schizolobium amazonicum Huber ex Ducke and Sclerolobium paniculatum Vogel (Caesalpiniaceae) and its importance in forestry management projects. In: The International Pollination Simposium, Lethbridge. Acta Horticulturae 437: 65-69.

Vogel, S. 1954. Blütenbiologische Typen als Elemente der Sippengliederung, dargestellt anhand der Flora Südafrikas. Botanische Studien 1:1-338.

Wallace, H. 1981. An assessment of diet overlap indexes. Transactions of the American Fisheries Society 110: 72-76.

Wille, A. 1963 Behavioral adaptations of bees for pollen collecting from Cassia flowers. Revista de Biologia Tropical 11: 205-210.

Willmer, P; D. Stanley; K. Steijven; I. Matthews \& C. Nuttman. 2009. Bidirectional flower color and shape changes allow a second opportunity for pollination. Current Biology 19: 919-923.

Zaret T. M. \& A. S. Rand. 1971. Competition in tropical stream fishes: support for the competitive exclusion principle. Ecology 52: 336-342.

Received 24/8/2010; accepted 19/4/2011

Editor: Maria Cristina Gaglianone 